\title{
Reuse of liver allograft from a brain-dead recipient: a case report
}

\author{
Min-Jae Kim, Shin Hwang, Dong-Hwan Jung, Gil-Chun Park, Gi-Won Song, Hwui-Dong Cho, Sung-Gyu Lee
}

Department of Surgery, Asan Medical Center, University of Ulsan College of Medicine, Seoul, Korea

Background: We report our first case of deceased-donor liver transplantation (LT) using a reuse liver graft after the first LT. Methods: The recipient was a 38-year-old female with fulminant hepatic failure from toxic hepatitis.

Results: She had a history of herb intake and her liver function deteriorated progressively. She was enrolled as the Korean Network for Organ Sharing (KONOS) status 1 and the model for end-stage liver disease score was 34. The donor was a 42-year-old male patient who fell into brain death after LT for alcoholic liver cirrhosis. Donation of multiple organs including the transplanted liver graft was performed 10 days after the first LT operation. Since the liver graft appeared to be normal and frozen-section liver biopsy showed only mild fatty changes, we decided to reuse the liver graft. A modified piggy-back technique of the suprahepatic inferior vena cava reconstruction was used. Other surgical procedures were comparable to the standard deceased-donor LT procedures. The explant liver pathology revealed submassive hepatic necrosis, which was compatible with toxic hepatitis. The peak of serum liver enzyme levels were aspartate transaminase 1,063 IU/L and alanine transaminase $512 \mathrm{IU} / \mathrm{L}$ at posttransplant day 3. Since the pretransplant general condition of the recipient was very poor, hospital stay was prolonged and she was discharged 51 days after LT operation. She is currently doing well for 3 years to date.

Conclusions: Experience in our case and the literature review suggest that a reuse liver graft can be regarded as one of the marginal grafts which can be transplantable to the LT candidates requiring urgent LT.

Corresponding author: Shin Hwang

E-mail: shwang@amc.seoul.kr

(c) The Korean Society for Transplantation

This is an Open Access article distributed under the terms of the Creative Commons Attribution Non-Commercial License (http://creativecommons.org/licenses/by-nc/4.0/) which permits unrestricted non-commercial use, distribution, and reproduction in any medium, provided the original work is properly cited. 